\title{
Investigation on the Cognitive Situation of Gargles by Dentists with Different Titles in a Province of China: A Cross-sectional Survey
}

\author{
Na Liu ${ }^{\dagger}$, Yue Liu ${ }^{\dagger}$, Hailin Zhang, Qing Liu* \\ School and Hospital of Stomatology, Hebei Medical University \& Hebei Key Laboratory of Stomatology, Shijiazhuang, China \\ Email address: \\ 2036369127@qq.com (Na Liu), 292837237@qq.com (Yue Liu), helen96@126.com (Hailin Zhang),656069017@qq.com (Qing Liu) \\ *Corresponding author \\ $\uparrow \mathrm{Na}$ Liu and Yue Liu are co-first authors.
}

To cite this article:

$\mathrm{Na}$ Liu, Yue Liu, Hailin Zhang, Qing Liu. Investigation on the Cognitive Situation of Gargles by Dentists with Different Titles in a Province of China: A Cross-sectional Survey. Clinical Medicine Research. Vol. 9, No. 3, 2020, pp. 47-53. doi: 10.11648/j.cmr.20200903.11

Received: May 8, 2020; Accepted: May 26, 2020; Published: June 3, 2020

\begin{abstract}
Background and objective: We found that the phenomenon of unreasonable use of gargles is common in clinical practice, which is closely related to the lack of recognition of gargles by dentists. In this study, some dentists in Hebei province of China were investigated to find some problems with their cognition and some reasonable suggestions were put forward. Methods: 600 dentists were chosen by stratified random sampling. A self-designed questionnaire was distributed online to study the knowledge of those dentists with different professional titles on the gargles types, drug resistance, adverse reactions, potential risks and anti-Candida albicans. 576 questionnaires were collected, of which 528 were valid. All valid questionnaires were analyzed by SPSS21.0 software. Results: As for the gargle types, doctors who knew nothing about it accounted for $15.15 \%$. $51.52 \%$ of the dentists did not pay attention to the drug resistance. As for the adverse reactions of compound chlorhexidine gargle, doctors who chose "no adverse reactions" and "unclear" accounted for $8.71 \%$ and $32.58 \%$ respectively. The awareness of "taste change" of primary dentists was lower than that of senior dentists $(\mathrm{P}<0.05)$. As for the potential risks of long-term and frequent use of gargles, $87.50 \%$ of doctors chose "Oral flora disorder". Doctors who chose other risks were relatively few accounting for $6.44 \%$, and those who chose "unclear" accounted for $12.12 \%$. As for the cognition of gargles against Candida albicans, $7.58 \%$ of dentists chose "unclear". Conclusion: The overall cognition of gargles by dentists with different titles is not comprehensive or accurate.
\end{abstract}

Keywords: Dentists, Gargles, Cognition, Survey

\section{Introduction}

Gargles are commonly used therapeutic drug for adjuvant treatment of such oral diseases as periodontal disease, caries, and oral mucosal disease. They have good effects in eliminating oral odor, reducing oral bacteria, preventing dental caries, anti-inflammatory and analgesic [1-4], which has increasingly been recognized by dentists and patients. However, as prescription drugs, they have strict indications and contraindications, and their side effects cannot be ignored. The full cognition of gargles by a professional dentist is directly related to the patients' medication effect and safety.

In this study, a questionnaire survey was conducted among dentists in a province of China to preliminarily grasp the cognitive situation of dentists about gargles, and to analyze and discuss the results of the survey. By presenting relevant data, dentists were encouraged to further study the theoretical knowledge and spur the research development of gargles, thereby promoting the scientification of clinical use of them.

\section{Materials and Methods}

\subsection{Sample and Setting}

A cross-sectional survey was conducted in Hebei Province, China, from November 6-9, 2018. Stratified random sampling was used to select 600 study participants who were dentists in stomatology department of general hospitals, dental specialty 
hospitals, community hospitals, dental clinics in Hebei Province of China. We stratified the sample by titles: senior title, intermediate title, primary title and ungraded. To be eligible their working experience needs to be over one year. Stomatology educators responsible for stomatological management/teaching were excluded.

\subsection{Design and Contents of the Questionnaire}

The questionnaire includes 11 questions, with 6 single-choice questions and 5 multiple-choice questions and consists of two parts: 1 . basic information of the dentists, including the name, level and major, as well as the title and age of the respondents. 2. gargles-related knowledge of dentists, including types, drug resistance, adverse reactions, potential risks, and the killing of Candida albicans. Face validity was obtained by three professional dentists reviewing a draft questionnaire. The questionnaire was made available online as a web-based link and tried out with the participants. No formal pilot study of the questionnaire was performed. The responding reported no significant issues, so the questionnaire was considered acceptable and a link to the final version was emailed to the target participants. Incomplete questionnaires were excluded to preserve the accuracy of the analysis.

\subsection{Data Collection}

An online questionnaire survey was distributed to dentists. The online survey was performed via e-mails that contained a link to an online questionnaire, which were sent to the e-mail addresses of the targeted hospitals or clinics. The survey respondents were anonymous and not linked to the submitting source, so that response rates were not known by the original disseminated e-mail addresses. The questionnaire was accompanied by detailed instructions. If there was any doubt in the filling process, the respondents could get any help and explanations by the investigators. In order to minimize communicating between the subjects, the questionnaire system was only open for 72 hours.

\subsection{Statistical Method}

Statistical Program for Social Sciences 21.0 software (SPSS21.0, Inc., Chicago, IL, USA) was used to analysis the data. $\chi^{2}$ test or Fisher exact probability method was used to compare the cognition of gargles among dentists with different professional titles. The test level was $\alpha=0.05, P<0.05$. The difference was statistically significant.

\subsection{Ethical Clearance}

This study had been approved by the ethics committee of Stomatology Hospital of Hebei Medical University, and we confirmed that informed consents had been obtained from all participants. If the responders feel that the survey has a negative impact on their work or life, they have the right to withdraw from the survey at any time.

\section{Results}

\subsection{General Situation}

A total of 576 questionnaires were collected, among which, 48 incomplete questionnaires were excluded and 528 were valid. The response rate was $96 \%$ and effective rate was $91.67 \%$. A majority of respondents were from hospital $(n=464$, $87.87 \%)$. Most respondents were under $40(n=414,78.41 \%)$ (Table 1). Among the respondents, 128 held senior professional titles, accounting for $24.34 \%, 154$ intermediate titles, $29.17 \%$, 146 junior titles, $27.65 \%$, and 100 were ungraded, 18.94\% (Figure 1, Table 1).

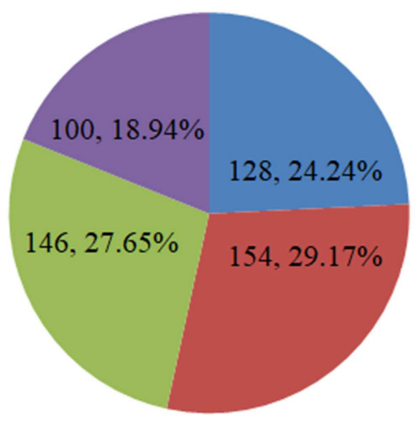

घenior $\square$ Intermediate $\quad$ Primary $\square$ Ungraded

Figure 1. Professional Title Distribution of dentists.

Table 1. Characteristics of respondents.

\begin{tabular}{llll}
\hline & & $\mathbf{N}=\mathbf{5 2 8}$ & $\mathbf{\%}$ \\
\hline \multirow{3}{*}{ Hospital } & General hospital & 256 & 48.48 \\
& Stomatological Hospital & 208 & 39.39 \\
& Clinic & 64 & 12.12 \\
\multirow{4}{*}{ Age } & $<=30$ & 236 & 44.7 \\
& $31-40$ & 178 & 33.71 \\
& $41-50$ & 96 & 18.18 \\
\multirow{4}{*}{ Title } & $>50$ & 18 & 3.14 \\
& Senior title & 128 & 24.24 \\
& Intermediate title & 154 & 29.17 \\
& Primary & 146 & 27.65 \\
& Ungraded & 100 & 18.94 \\
\hline
\end{tabular}

\subsection{Cognitive Situation of Gargle Types}

Gargles are mainly used in the treatment of the periodontal disease and oral mucosal disease, and in the prevention of caries. 400 dentists knew a lot about the types of gargles for treating the periodontal disease, accounting for $75.76 \%$, followed by 80 dentists who knew little about the types of gargles for treating the oral mucosal disease and preventing the caries, accounting for $15.15 \%$. There was no statistical significance in the perception of gargle types among dentists with different professional titles $(P>0.05)$ (Table 2). 
Table 2. Cognition of gargle types by dentists with different professional titles.

\begin{tabular}{|c|c|c|c|c|c|c|c|}
\hline Gargle types & $\begin{array}{l}\begin{array}{l}\text { Senior } \\
(\mathrm{n}=128)\end{array} \\
\end{array}$ & $\begin{array}{l}\text { Intermediate } \\
(\mathrm{n}=154)\end{array}$ & $\begin{array}{l}\text { Primary } \\
(n=146)\end{array}$ & $\begin{array}{l}\text { Ungraded } \\
(\mathrm{n}=100)\end{array}$ & $\begin{array}{l}\text { Total } \\
(\mathrm{n}=528)\end{array}$ & \multirow[t]{2}{*}{$\chi^{2}$} & \multirow[t]{2}{*}{$\boldsymbol{P}$} \\
\hline & Choice (\%) & Choice (\%) & Choice (\%) & Choice (\%) & Choice (\%) & & \\
\hline Treatment of periodontal disease & $100(78.13)$ & $122(79.22)$ & $110(75.34)$ & $68(68.00)$ & $400(75.76)$ & 4.69 & 0.20 \\
\hline Treatment of Mucosal Disease & $42(32.18)$ & $54(35.06)$ & $52(35.62)$ & $34(34.00)$ & $182(34.47)$ & 0.28 & 0.97 \\
\hline Caries prevention & $24(18.75)$ & $28(18.18)$ & $26(17.81)$ & $22(22.00)$ & $100(18.94)$ & 0.79 & 0.85 \\
\hline None of the above & $16(12.50)$ & 20 (12.99) & $26(17.81)$ & $18(18.00)$ & $80(15.15)$ & 2.69 & 0.44 \\
\hline
\end{tabular}

\subsection{Concerns Situation About Drug Resistance of Gargles}

Among the 528 dentists, 256 claimed to have paid attention to the drug resistance of gargle, accounting for $48.48 \%$, whereas 272 claimed to have not paid attention to it, accounting for $51.52 \%$, the proportion of the former being lower than that of the latter. The proportion of senior dentists who were concerned about gargle resistance was $68.75 \%$, which was higher than the proportion of the unconcerned dentists, standing at $31.25 \%$, but the proportion of dentists with intermediate, primary and ungraded titles who were concerned about gargle resistance was lower than that of those unconcerned dentists.

The proportion of senior dentists who were concerned about gargle resistance was significantly higher than that of those dentists with intermediate, primary and undetermined dentists $(P<0.01)$. There was no statistical significance in the proportion among the latter three groups of the dentists regarding gargle resistance $(P>0.05)$ (Table 3$)$.

Table 3. Concerns of dentists with different professional titles on gargle resistance.

\begin{tabular}{|c|c|c|c|c|c|c|c|}
\hline Attention & Senior (\%) & Intermediate (\%) & Primary (\%) & Ungraded (\%) & Total (\%) & $\chi^{2}$ & $P$ \\
\hline concerned & $88(68.75)$ & $64(41.56) *$ & $62(42.47)^{*}$ & $42(42.00)^{*}$ & $256(48.48)$ & & \\
\hline Unconcerned & $40(31.25)$ & $90(58.44)$ & $84(57.53)$ & $58(58.00)$ & $272(51.52)$ & 27.81 & $<0.01$ \\
\hline Total & $128(24.24)$ & $154(29.17)$ & $146(27.65)$ & $100(18.94)$ & 528 & & \\
\hline
\end{tabular}

Note: *: Compared with senior dentists, $P<0.05$.

\subsection{Cognition of Adverse Reactions of Gargle}

The most commonly used Compound Chlorhexidine Gargle was selected to investigate the cognition of dentists on the adverse reactions of gargles. The results showed that, as for the adverse reactions, the number of dentists who were aware of tooth staining, tongue coating yellow and taste changes were relatively higher, accounting for $51.52 \%, 43.18 \%$ and $41.67 \%$ respectively, and the awareness rate of other adverse reactions was lower than $25 \%$. There were 46 dentists who chose "no adverse reaction" and 172 dentists who chose "unclear", accounting for $8.71 \%$ and $32.58 \%$ respectively.

The awareness of "tooth staining" among senior dentists was significantly higher than that among intermediate, primary and ungraded dentists $(P<0.05)$. As for "taste change", the awareness of ungraded dentists was significantly lower than that of senior, intermediate and junior dentists $(P<0.05)$. The awareness of "taste change" among primary dentists was lower than that of senior dentists $(\mathrm{P}<0.05)$. As for other adverse reactions, there was no statistical significance among dentists with different professional titles $(P>0.05)$ (Table 4).

Table 4. The perception of adverse reactions of gargles among dentists with different professional titles.

\begin{tabular}{|c|c|c|c|c|c|c|c|}
\hline \multirow[t]{2}{*}{ Adverse reaction } & $\begin{array}{l}\text { Senior } \\
(n=128)\end{array}$ & $\begin{array}{l}\text { Intermediate } \\
(n=154)\end{array}$ & $\begin{array}{l}\text { Primary } \\
(n=146)\end{array}$ & $\begin{array}{l}\text { Ungraded } \\
(n=100)\end{array}$ & $\begin{array}{l}\text { Total } \\
(n=528)\end{array}$ & \multirow[t]{2}{*}{$\chi^{2}$} & \multirow[t]{2}{*}{$\boldsymbol{P}$} \\
\hline & Choice (\%) & Choice (\%) & Choice (\%) & Choice (\%) & Choice (\%) & & \\
\hline Tooth staining & $80(62.50)$ & $72(46.75)^{*}$ & $74(50.68)^{*}$ & $46(46.00)^{*}$ & $272(51.52)$ & 8.84 & 0.03 \\
\hline Yellowing of tongue coating & $54(42.19)$ & $64(41.56)$ & $66(45.21)$ & $44(44.00)$ & $228(43.18)$ & 0.49 & 0.92 \\
\hline Dysgeusia & $64(50.00)$ & $66(42.86)$ & $60(41.10)$ & $30(30.00) * \# \Delta$ & $220(41.67)$ & 45.25 & $<0.01$ \\
\hline Dry & $30(23.44)$ & $24(15.58)$ & $40(27.40)$ & $24(24.00)$ & $118(22.35)$ & 6.45 & 0.09 \\
\hline Metallic odor in oral cavity & $24(18.75)$ & $20(12.99)$ & $28(19.18)$ & $20(20.00)$ & $92(17.42)$ & 3.04 & 0.39 \\
\hline Allergic reaction & $38(29.69)$ & $44(28.57)$ & $24(16.44)$ & $20(20.00)$ & $126(23.86)$ & 0.36 & 0.55 \\
\hline No adverse reaction & $6(4.69)$ & $12(7.79)$ & $14(9.59)$ & $14(14.00)$ & $46(8.71)$ & 6.16 & 0.10 \\
\hline Unclear & $36(28.13)$ & $44(28.57)$ & $54(36.99)$ & $38(38.00)$ & $172(32.58)$ & 4.91 & 0.18 \\
\hline
\end{tabular}

Note: *: Compared with senior dentists, $P<0.05$; \#: Compared with intermediate dentists, $\mathrm{P}<0.05$; $\Delta$ : Compared with junior dentists, $P<0.05$.

\subsection{Cognition of Potential Risks of Long-term Frequent Use of Gargles}

According to the survey, as for the most potential risk of frequent use of gargles for a long time,, the number of dentists who chose "Oral flora disorder" was the highest among the respondents, accounting for $87.50 \% .12 .12 \%$ of dentists were "unclear". Only $6.44 \%$ of dentists knew about "elevated blood pressure risk" and "increased risk of neck lymphoma".

Dentists with ungraded professional titles had a poorer understanding of risks. And the proportion of dentists who chose "unclear" was significantly higher than that of dentists with the other three professional titles $(P<0.05)$. As for "oral 
flora disorder", the perception of dentists with ungraded professional titles was significantly lower than that of dentists with senior, intermediate and junior professional titles $(P<0.05)$. As for other risks, there was no statistical significance in the perception of dentists with different professional titles (Fisher exact probability method, $P>0.05$ ) (Table 5).

Table 5. Cognition of potential risks of gargle by dentists with different professional titles.

\begin{tabular}{|c|c|c|c|c|c|c|c|}
\hline \multirow[t]{2}{*}{ Potential risks } & $\begin{array}{l}\text { Senior } \\
(n=128)\end{array}$ & $\begin{array}{l}\text { Intermediate } \\
(n=154)\end{array}$ & $\begin{array}{l}\text { Primary } \\
(n=146)\end{array}$ & $\begin{array}{l}\text { Ungraded } \\
(n=100)\end{array}$ & $\begin{array}{l}\text { Total } \\
(n=528)\end{array}$ & \multirow[t]{2}{*}{$\chi^{2}$} & \multirow[t]{2}{*}{$\boldsymbol{P}$} \\
\hline & Choice (\%) & Choice (\%) & Choice (\%) & Choice (\%) & Choice (\%) & & \\
\hline Oral flora disorder & $116(90.63)$ & $138(89.61)$ & $134(91.78)$ & $74(74.00) * \# \Delta$ & $462(87.50)$ & 20.88 & $<0.01$ \\
\hline Increasing blood pressure risk & $4(3.13)$ & $6(3.90)$ & $6(4.11)$ & $4(4.00)$ & $20(3.79)$ & 0.32 & 0.98 \\
\hline Increased risk of neck lymphoma & $4(3.13)$ & $2(1.30)$ & $6(4.11)$ & $2(2.00)$ & $14(2.65)$ & 2.49 & 0.47 \\
\hline Unclear & $8(6.25)$ & $20(12.99)$ & $12(8.22)$ & $24(24.00) * \# \Delta$ & $64(12.12)$ & 19.58 & $<0.01$ \\
\hline
\end{tabular}

Note: *: Compared with senior dentists, $P<0.05$; \#: Compared with intermediate dentists, $\mathrm{P}<0.05 ; \Delta$ : Compared with junior dentists, $P<0.05$.

\subsection{Cognition of the Anti-Candida Albicans Effect of Gargles}

As for compound chlorhexidine gargle, cetylpyridinium chloride gargle and povidone gargle, $7.58 \%$ of the dentists were not sure whether they had the effect of anti-Candida albicans. Among the three gargles, only $54.92 \%$ of the dentists had a relatively high awareness of the effect of compound chlorhexidine gargle.
Compared with senior professional dentists, intermediate and junior professional dentists had a higher awareness of the effect of compound chlorhexidine gargle and cetylpyridinium chloride gargle on Candida albicans $(P<0.01)$. There was no statistical significance in the difference in cognition of povidone gargle against Candida albicans among all groups $(P>0.05)$ (Table 6).

Table 6. Cognition of the anti-Candida albicans effect of gargle by dentists with different professional titles.

\begin{tabular}{|c|c|c|c|c|c|c|c|}
\hline \multirow[t]{2}{*}{ Gargle types } & $\begin{array}{l}\text { Senior } \\
(n=128)\end{array}$ & $\begin{array}{l}\text { Intermediate } \\
(n=154)\end{array}$ & $\begin{array}{l}\text { Primary } \\
(n=146)\end{array}$ & $\begin{array}{l}\text { Ungraded } \\
(n=100)\end{array}$ & $\begin{array}{l}\text { Total } \\
(n=528)\end{array}$ & \multirow[t]{2}{*}{$\chi^{2}$} & \multirow[t]{2}{*}{$\boldsymbol{P}$} \\
\hline & Choice (\%) & Choice (\%) & Choice (\%) & Choice (\%) & Choice (\%) & & \\
\hline Compound chlorhexidine gargle & $56(43.75)$ & $94(61.04)^{*}$ & $92(63.01)^{*}$ & $48(48.00)$ & $290(54.92)$ & 14.58 & $<0.01$ \\
\hline Cetylpyridinium chloride gargle & $24(18.75)$ & $52(33.77)^{*}$ & $56(38.36)^{*}$ & $24(24.00)$ & $156(29.55)$ & 15.41 & $<0.01$ \\
\hline Povidone gargle & $24(18.75)$ & $18(11.69)$ & $28(19.18)$ & $12(12.00)$ & $82(15.53)$ & 5.18 & 0.16 \\
\hline Unclear & $8(6.25)$ & $8(5.19)$ & $12(8.22)$ & $12(12.00)$ & $40(7.58)$ & 4.45 & 0.22 \\
\hline
\end{tabular}

Note: *: Compared with senior dentists, $\mathrm{P}<0.05$.

\section{Discussions and Conclusion}

It is self-evident that the rational use of mouthwash, an auxiliary drug in oral clinic, is important. The premise of rational use is that dentists have a comprehensive understanding of gargles. One-sided understanding will inevitably lead to wrong selection of indications, increased incidence of drug resistance, adverse reactions and so on. Therefore, this study has a strong practical significance in promoting dentists to learn the theory of gargles and enhancing the scientificalization of clinical medication of gargles through the investigation of dentists' perception of them.

There are wide varieties of gargles, including antibiotics, surfactants, traditional Chinese medicines and a few ethnic herbs. As far as their functions are concerned, gargles can prevent periodontal diseases, oral ulcers, caries, etc. There are prescriptions and non-prescriptions as for the usage [5-11]. The mouth rinses for periodontal disease, oral mucosal disease and caries prevention types listed in this survey are more common gargles. From the survey results, periodontal disease treatment gargle is the most familiar to dentists. According to the results of the fourth national oral health epidemiological survey, the periodontal health rates of the 35-44 age group,
55-64 age group and 65-74 age group in China are only $9.1 \%$, $5.0 \%$ and $9.3 \%$ [12]. The national periodontal problems are aggravating, which inevitably leads to the increase of prescription rate of periodontal treatment gargles, and dentists have a relatively high understanding of it. However, we should also clearly see that $15.15 \%$ of the dentists do not understand all kinds of gargles. These dentists have a large blindness in the prescription of gargles, and it is even more difficult to scientifically and reasonably guide patients how to use gargles. In addition, there was no statistical significance among the dentists with different professional titles $(P>0.05)$, which indicates that dentists do not have the corresponding enhancement of theoretical knowledge related to gargles with the growth of their qualifications in clinical work.

Resistance of bacteria to antibiotics is a major issue in clinical medicine in recent years, and the level of bacterial resistance is increasing [10,13-15]. At present, almost all bacteria can acquire different resistance genes, and each antibiotic can be resisted and destroyed by some bacteria to varying degrees [16]. With the increasing use of antibiotics, most strains may show resistance to more than one antibiotic. Anaerobic bacteria in oral and maxillofacial infections have a high resistance rate to metronidazole [17]. As a prescription antibiotic, gargles also have the risk of drug resistance. For 
instance, the most commonly used compound chlorhexidine gargle in oral clinic contains metronidazole, which may lead to bacterial resistance if it is improperly used. Dentists should have a clear understanding of the risk. However, the survey showed that $51.52 \%$ of dentists did not pay attention to the problem of gargles resistance and only senior dentists paid a little higher attention to gargles resistance, which only accounted for $68.75 \%$. Literature researches were done with the keywords "gargles" or "mouthwash" or "mouth rinse", "drug resistance", and no relevant research reports or literatures were retrieved. From a subjective point of view, the dentists did not take the initiative to pay attention to the problem of gargle resistance. Whereas objectively, there is little information about gargles resistance, so it is difficult for dentists to obtain it.

Different gargles have different adverse reactions, which are usually marked on the instructions. For example, for the most commonly used compound chlorhexidine gargle, adverse reactions include tooth staining, yellowing of tongue coating, taste changes, etc. In addition, other side effects, such as dry mouth, astringent mouth, numbness of upper lip, itching of lips, slight tingling of oral mucosa and so on, may be caused by compound chlorhexidine gargle when used locally in the mouth because metronidazole is an effective component of it [18]. Besides, Lei Zhaobao and others considered that adverse reactions still include contact dermatitis and serious cases which can produce anaphylactic shock or even sudden death $[19,20]$. Other gargles may also have these adverse reactions such as cetylpyridinium chloride gargle, whose main active ingredient is cetylpyridinium chloride. Although the adverse reactions labeled in the instructions are not clear, occasional taste disorders have been reported. The mechanism may be related to drug-induced zinc deficiency in the body, its own taste, damages to taste cells or taste bud centers, changes in chemical composition of saliva and mucus and the sensory information process of taste $[21,22]$. The survey took compound chlorhexidine gargle as an example and some adverse reactions in the manual and literature were listed to give dentists a choice. The results showed that the knowledge rate of dentists on tooth coloring, tongue coating yellow and taste changes was relatively high, but only accounting for $51.25 \%, 43.18 \%$, and $41.67 \%$, respectively. Professional dentists at all levels do not have a comprehensive understanding of adverse reactions, few dentists made the right choice, and senior professional dentists are no better than other professional titles. It is worth noting that 218 people choose "no adverse reactions" and "unclear", which means that about $41.29 \%$ of the dentists had wrong knowledge or did not know about the adverse reactions of gargles, and among the dentists who did not choose the two items, there were still cognitive incompleteness. The accuracy and comprehensiveness of cognition are worrying. It is suggested that dentists at all levels should read the instructions carefully and strengthen literature reading.

Gargle will produce some side effects if it is used frequently for a long time. Therefore, it should be used under the guidance of dentists, and cannot be used for a long time. There is normal flora in healthy oral cavity, and long-term use of gargles with bactericidal effect will lead to oral flora imbalance. Some studies have found that the use of anti-bacterial gargles can destroy oral flora and cause stress reaction, which can increase blood pressure, and has the potential to bring adverse cardiovascular diseases to healthy population $[23,24]$. Studies have also shown that frequent use of gargle over a long period of time increases the potential risk of head and neck cancer [25]. Dentists' attentions are infrequently paid to this regard, most of which is limited to flora imbalance, and little is paid to other rare side effects. Even $12.12 \%$ of dentists do not know what potential adverse effects the frequent use of gargles will bring to patients. Although these conclusions need to be further studied and confirmed by evidence-based medicine, dentists should pay attention to the latest research trends of gargles.

Candida albicans is one of the most common symbiotic bacteria in oral cavity. With the application of antibiotics and the development of radiation medicine, the normal flora of oral cavity is imbalanced and Candida albicans multiplies in large numbers and causes disease [26-28]. Some gargles have scavenging effect on Candida albicans. Some studies have shown that compound chlorhexidine gargle and $0.5 \%$ povidone iodine gargle have the same bacteriostatic effect on Candida albicans, but significantly higher than $0.1 \%$ cetylpyridinium chloride gargle [29]. Some experiments also showed that $0.1 \%$ cetylpyridinium chloride gargle had a certain effect on oral Candida infection. There was no statistical significance between $0.1 \%$ cetylpyridinium chloride gargle and $1 \%$ povidiolone in improving clinical symptoms and fungal clearance rate, or cetylpyridinium chloride gargle was slightly dominant [30, 31]. Regardless of the intensity of the action, compound chlorhexidine gargle, cetylpyridinium chloride gargle and povidone gargle had the killing effect on Candida albicans. According to the questionnaire data, 54.92\% of the dentists chose the compound chlorhexidine gargle, and nearly half of the dentists did not know it, while dentists had a very low understanding on the effect of killing Candida albicans of the other two mouthwashes, accounting for $7.58 \%$. This shows that dentists have a one-sided understanding of how to kill Candida albicans with gargles.

In conclusion, the overall cognition of gargles by dentists in this province is not comprehensive or accurate, and the senior dentists do not show a higher level of cognition in general. This is a sample survey, but it can also be inferred that the overall perception of gargles among all dentists in China is not very optimistic. Error and one-sided cognition seriously affect the efficacy and safety of gargles. Based on that, we put forward to the following suggestions to Chinese dentists and relevant departments, which may be of reference value to the rest of the world: 1. Basic and clinical researches on gargles should be further carried out, not only to study the mechanism and efficacy, but also to study drug resistance, adverse reactions and side effects, so as to continuously enrich the relevant theories of gargles. 2. The active learning of dentists should be further strengthened and literature reading should not be overlooked at any stage of their practice. They need to 
continue to learn the various theories about gargles while keeping a close eye on the frontiers and trends of gargles researches. 3. Continuing medical education and other forms should be taken to promote dentists to learn the relevant theories of gargles, and it is necessary to realize the dynamic adjustment of the prescription right through the regular assessment of dentists.

\section{Acknowledgements}

This study was conducted with the support of Government-funded Training Project for Specialist Leaders in Hebei Provincial in China and Innovative Experimental Project for College Students in Hebei Province in China. We would like to thank Hebei Provincial Finance Department and Hebei Medical University.

\section{References}

[1] Blom T, Slot DE, Quirynen M, et al. The effect of mouthrinses on oral malodor: a systematic review. International Journal of Dental Hygiene, 2012, 10: 209-22.

[2] Marsh PD. Contemporary perspective on plaque control. Br Dent J, 2012, 212: 601-6.

[3] Prasad M, Patthi B, Singla A, et al. The Clinical Effectiveness of Post-Brushing Rinsing in Reducing Plaque and Gingivitis: A Systematic Review. J Clin Diagn Res, 2016, 10: E1-7.

[4] Zero DT. Dentifrices, mouthwashes, and remineralization/caries arrestment strategies. BMC Oral Health, 2006, 6 Suppl 1: S9.

[5] Albert-Kiszely A, Pjetursson BE, Salvi GE, et al. Comparison of the effects of cetylpyridinium chloride with an essential oil mouth rinse on dental plaque and gingivitis - a six-month randomized controlled clinical trial. JOURNAL OF CLINICAL PERIODONTOLOGY, 2007, 34: 658-67.

[6] Barnett ML. The rationale for the daily use of an antimicrobial mouthrinse. JOURNAL OF THE AMERICAN DENTAL ASSOCIATION, 2006, 137 Suppl: 16S-21S.

[7] Eslami H, Pouralibaba F, Falsafi P, et al. Efficacy of Hypozalix spray and propolis mouthwash for prevention of chemotherapy-induced oral mucositis in leukemic patients: A double-blind randomized clinical trial. J Dent Res Dent Clin Dent Prospects, 2016, 10: 226-33.

[8] Hwang BY, Roberts SK, Chadwick LR, et al. Antimicrobial constituents from goldenseal (the Rhizomes of Hydrastis canadensis) against selected oral pathogens. PLANTA MEDICA, 2003, 69: 623-7.

[9] Marinho VC, Higgins JP, Sheiham A, et al. Combinations of topical fluoride (toothpastes, mouthrinses, gels, varnishes) versus single topical fluoride for preventing dental caries in children and adolescents. Cochrane Database Syst Rev, 2004: D2781.

[10] Palmer KL, Kos VN, Gilmore MS. Horizontal gene transfer and the genomics of enterococcal antibiotic resistance. CURRENT OPINION IN MICROBIOLOGY, 2010, 13: 632-9.

[11] Van der Weijden FA, Van der Sluijs E, Ciancio SG, et al. Can Chemical Mouthwash Agents Achieve Plaque/Gingivitis
Control? Dent Clin North Am, 2015, 59: 799-829.

[12] Lu HX, Tao DY, Lo E, et al. The 4th National Oral Health Survey in the Mainland of China: Background and Methodology 2018.

[13] Frere JM, Sauvage E, Kerff F. From "An Enzyme Able to Destroy Penicillin" to Carbapenemases: 70 Years of Beta-lactamase Misbehaviour. CURRENT DRUG TARGETS, 2016, 17: 974-82.

[14] Sharma VK, Johnson N, Cizmas L, et al. A review of the influence of treatment strategies on antibiotic resistant bacteria and antibiotic resistance genes. CHEMOSPHERE, 2016, 150: 702-14.

[15] Yong D, Toleman MA, Giske CG, et al. Characterization of a new metallo-beta-lactamase gene, bla (NDM-1), and a novel erythromycin esterase gene carried on a unique genetic structure in Klebsiella pneumoniae sequence type 14 from India. Antimicrob Agents Chemother, 2009, 53: 5046-54.

[16] Martinez JL. General principles of antibiotic resistance in bacteria. Drug Discov Today Technol, 2014, 11: 33-9.

[17] Bao ZY, Lin Q, Meng YH, et al. [Application of anaerobic bacteria detection in oral and maxillofacial infection]. Beijing Da Xue Xue Bao Yi Xue Ban, 2016, 48: 76-9.

[18] Autio-Gold J. The role of chlorhexidine in caries prevention. OPERATIVE DENTISTRY, 2008, 33: 710-6.

[19] Ebo DG, Bridts CH, Stevens WJ. IgE-mediated anaphylaxis from chlorhexidine: diagnostic possibilities. CONTACT DERMATITIS, 2006, 55: 301-2.

[20] Lim KS, Kam PC. Chlorhexidine--pharmacology and clinical applications. Anaesth Intensive Care, 2008, 36: 502-12.

[21] Kulkarni VV, Damle SG. Comparative evaluation of efficacy of sodium fluoride, chlorhexidine and triclosan mouth rinses in reducing the mutans streptococci count in saliva: an in vivo study. J Indian Soc Pedod Prev Dent, 2003, 21: 98-104.

[22] Naik BS, Shetty N, Maben EV. Drug-induced taste disorders. European Journal of Internal Medicine, 2010, 21: 240-3.

[23] Atala MH, Ustaglu G, Atala N, et al. Effect of different mouthwashes on discoloration of plaque-free tooth surfaces. AMERICAN JOURNAL OF DENTISTRY, 2018, 31: 211-4.

[24] Delgado AJ, Dias RA, Quesada A, et al. Potential erosive effect of mouthrinses on enamel and dentin. Gen Dent, 2018, 66: $75-9$.

[25] Boffetta P, Hayes RB, Sartori S, et al. Mouthwash use and cancer of the head and neck: a pooled analysis from the International Head and Neck Cancer Epidemiology Consortium. EUROPEAN JOURNAL OF CANCER PREVENTION, 2016, 25: 344-8.

[26] Gulati M, Nobile CJ. Candida albicans biofilms: development, regulation, and molecular mechanisms. MICROBES AND INFECTION, 2016, 18: 310-21.

[27] Lohse MB, Gulati M, Johnson AD, et al. Development and regulation of single- and multi-species Candida albicans biofilms. NATURE REVIEWS MICROBIOLOGY, 2018, 16: 19-31.

[28] Pappas PG, Rex JH, Sobel JD, et al. Guidelines for treatment of candidiasis. CLINICAL INFECTIOUS DISEASES, 2004, 38: 161-89. 
[29] Xu LL, Wang GQ, Ge YQ, et al. Clinical Observation on Bacteriostatic Effect of Four Kinds of Gargles on Candida Albicans. Journal of Nursing, 2015: 6-9.

[30] Wang Y. Contrasting analysis on effect of cetylpyridinium chloride and povidone iodine on oral candidiasis. Journal of Clinical Medicine in Practice, 2012, 16: 118-20.
[31] Herrera D, Escudero N, Perez L, et al. Clinical and microbiological effects of the use of a cetylpyridinium chloride dentifrice and mouth rinse in orthodontic patients: a 3-month randomized clinical trial. Eur J Orthod, 2018, 40: 465-74. 